\title{
Relación entre consumo de tabaco y alcohol y el autoconcepto en adolescentes españoles
}

\section{The relationship between consumption of tobacco and alcohol and self-concept in Spanish adolescents}

\author{
José Ignacio ÁLVARO GONZÁLEZ, Félix ZURITA ORTEGA, Manuel CASTRO \\ SÁNCHEZ, Asunción MARTÍNEZ MARTÍNEZ y Susana GARCÍA SÁNCHEZ \\ Universidad de Granada
}

Recibido: Septiembre 2014

Evaluado: Noviembre 2014

Aceptado: Noviembre 2014

\begin{abstract}
Resumen
La etapa adolescente constituye una fase esencial en el desarrollo de la persona; en estas edades las relaciones sociales y la adquisición de la personalidad son elementos claves, por tanto se planteó el siguiente estudio con los objetivos de determinar el autoconcepto de los adolescentes y cada una de sus dimensiones, así como describir el consumo habitual de alcohol y tabaco en la población adolescente y analizar las relaciones existentes entre el autoconcepto y las sustancias nocivas (alcohol y tabaco). Participaron un total de 2.134 adolescentes de la provincia de Granada (España), que permitió el registro y evaluación de las variables Autoconcepto (AF5), Consumo de Tabaco (FTND) y Consumo de Alcohol (AUDIT). Los resultados mostraron que el autoconcepto global era alto en los adolescentes, que en su mayoría eran no fumadores y el consumo de alcohol era perceptible únicamente en tres de cada diez jóvenes. De entre varias dimensiones las más puntuadas eran la familiar y la social. Se encontró asociación en el caso de las dimensiones de autoconcepto familiar, académico y social con el consumo de alcohol, sin embargo debe señalarse que el número de adolescentes que ingerían alcohol era bastante escueto por lo que debemos ser cautos con dicha afirmación.
\end{abstract}

Palabras clave: tabaco, alcohol, autoconcepto, adolescentes.

\begin{abstract}
The adolescent stage is an essential phase in the development of the individual; at this age social relations and the acquisition of personality are key elements, therefore the following study was proposed with the objectives of determining the self-concept of adolescents and each of its dimensions, as well as describing the habitual consumption of alcohol and tobacco in the adolescent population and to analyze the relationships between self-concept and harmful substances (alcohol and tobacco). A total of 2,134 adolescents from the province of Granada (Spain) participated in the study, which allowed the registration and evaluation of variables such as self-concept (AF5), tobacco consumption (FTND) and alcohol consumption (AUDIT). The results showed that global self-concept was high in those adolescents who in their majority were non-smokers, and alcohol consumption was only perceptible in three out of ten young


people. Among the highest ranked dimensions were the family and social ones. An association was found between the dimensions of familial, academic and social self-concept and alcohol consumption; however it should be noted that the number of adolescents who drank alcohol was quite short so we must be cautious with this statement.

Keywords: tobacco, alcohol, self-concept, adolescent.

La etapa adolescente representa una etapa esencial en el desarrollo del ser humano, pues en esta fase previa a la adultez, se halla caracterizada por no tener configurada y afianzada la personalidad, siendo fácilmente influenciables. Por ello es necesario transmitirles hábitos positivos y saludables, con el fin de sentar las bases en la adultez (Ruiz-Juan, De la Cruz, Ruiz-Ruisueño y García, 2008); ya que si durante su desarrollo no se adquieren los hábitos apropiados, posiblemente el ámbito cognitivo, físico y social (Carballo et al, 2013 y Villarreal-González, Sánchez y Musitu, 2013) se vean alterados.

Por ello, es determinante e imprescindible controlar los factores de riesgo que más fuerza ejercen sobre el adolescente, como son la familia, docentes, monitores deportivos y grupo de iguales, los cuales afianzan, apoyan o suprimen su patrón de conducta (Londoño, 2010; Ruiz-Juan, y Ruiz-Ruisueño, 2011). En este sentido, existen múltiples factores que inciden mutuamente unos sobre otros, y que terminan configurando la personalidad del adolescente. Una de las piezas clave de dicho engranaje es el autoconcepto. Éste se considera crucial en el desarrollo del individuo (Esnaola, Goñi y Madariaga, 2008), ya que se relaciona con diversos factores como son hábitos de vida, irregularidades en la alimentación, práctica deportiva, progreso en la vida, percepción de la dificultad, rendimiento, sexo y edad (Contreras, Fernández, García, Palou, y Ponseti, 2010; Soriano, Sampascual, y Navas, 2010).

El estudio del autoconcepto y su vínculo con el bienestar psicológico ha sido abordado por gran cantidad de autores del área científica en estas últimas décadas. Según Esnaola et al., (2008), se define como: "el conjunto de percepciones que una persona tiene de sí mismo"; en la década de los 70 Shavelson, Hubner, y Stanton (1976) establecieron las áreas del autoconcepto (afectiva, social, física, académica y personal), siendo el área física una de las más estudiadas, constituyéndose diferentes dimensiones para la misma, como habilidad física, condición física, atractivo físico y fuerza (Cox, 2009). Existen multitud de estudios que analizan este término, en ocasiones asociado a comportamientos violentos o agresivos (Jiménez, Musitu, Murgui y Lehalle, 2007; Cava, Murgui y Musitu, 2008; Jiménez, Musitu, Ramos y Murgui, 2009), a rendimiento escolar (Musitu y García, 2004), comunicación e influencia de tipo familiar (Estevez, Herrero, Martínez y Musitu, 2006; Jiménez et al., 2007) o práctica deportiva (Goñi e Infante, 2010; Esnaola, Rodríguez y Goñi, 2011; Gordillo, Vicente, Sánchez, Gómez y Gordillo, 2011), por lo que es necesario prestar la mayor atención posible a dichas variables para el correcto desarrollo del individuo en esta etapa de la adolescencia.

En este sentido, existe gran cantidad de autores que tratan de estudiar la relación existente entre el autoconcepto y el consumo de drogas, entre ellos señalamos a Echeburúa y Corral (2010) y Prado y Pantin (2011), los cuales afirman que un bajo 
autoconcepto, es uno de los principales factores de riesgo en el consumo de drogas. Sin embargo otros piensan que existen limitaciones en la medición del autoconcepto como un constructo unidimensional o multidimensional (Cava et al., 2008). Así, encontramos estudios muy variados en los que se utilizan medidas unidimensionales (Kavas, 2009 y Zamboaga, Schwartz, Jarvis y Van Tyne, 2009), que muestran como bajos niveles de autoconcepto, se corresponden con niveles elevados en el consumo de sustancias nocivas; otros no hallan un vínculo claro (Kokkevi, Richardson, Florescu, Kuzman y Stergar, 2007) y otros, aunque son minoría, postulan que un buen autoconcepto acarrea un consumo elevado de drogas (Oliva, Parra y Sánchez-Queija, 2008), aludiendo a causas sociales.

Por el contrario, hay una mayor diversidad en los resultados encontrados en el estudio de dicha relación basándose en medidas multidimensionales. Diferentes son las opiniones en función de la dimensión a tratar. En el caso de la dimensión social, algunos concluyen que los elementos sociales son inversamente proporcionales con la ingesta de sustancias (Téllez, Cote, Savogal, Martínez y Cruz, 2003) y otros piensan que aquellos individuos que se desenvuelven holgadamente en el área social mostrando facilidad en relacionarse con sus semejantes, tienen un perfil más adecuado para consumir drogas (Cava et al., 2008). En cuanto al ámbito familiar y académico parece estar claro que las familias que guían adecuadamente y dan buen ejemplo a sus condescendientes, propicia un mínimo consumo de sustancias perjudiciales por parte de éstos (Musitu, Jiménez y Murgui, 2007).

La práctica de actividad físico deportiva, no únicamente influye positivamente sobre la salud (Reigal, Videra, Parra y Juárez, 2012; González y Portolés (2014), sino que incide sobre otras variables en el ser humano, fundamentalmente en esta etapa, que son de gran importancia tales como el rendimiento académico, autoconcepto, motivación, consumo de alcohol, tabaco y drogas (Paz-Navarro, Roldán y González, 2009); de esta manera Esnaola et al., (2011) indican que la práctica de actividad física produce un rechazo hacia el consumo de alcohol y tabaco (drogas blandas).

Por todas estas razones, es necesario reforzar los aspectos que influyen sobre el autoconcepto desde las edades más tempranas, con objeto de que el adolescente lo desarrolle de la manera más adecuada; teniendo en cuenta estos antecedentes, se plantean los siguientes objetivos:

- Determinar el autoconcepto de los adolescentes y cada una de sus dimensiones.

- Describir el consumo habitual de alcohol y tabaco en la población adolescente.

- Analizar las relaciones existentes entre el autoconcepto y sus dimensiones, con el consumo de sustancias nocivas (alcohol y tabaco).

- Según estos objetivos se plantean tres hipótesis:

- El autoconcepto general de los adolescentes que consumen tabaco y alcohol de manera habitual es inferior a aquellos que no consumen.

- La ingesta frecuente de ambos elementos repercute negativamente en el autoconcepto de tipo Académico, Familiar y Físico.

- El tomar sustancias como tabaco y alcohol los fines de semana provoca un incremento en el Autoconcepto Social y Emocional. 
Este estudio nos reporta datos novedosos sobre el perfil de adolescentes granadinos concretamente en lo relacionado al autoconcepto y como la ingesta habitual de sustancias nocivas inciden en él, en una provincia y ciudad muy deprimida económicamente en la actualidad y donde no se ha detectado estudios recientes de estas características, si bien por la literatura consultada si se detecta que el inicio de estas prácticas de ingesta es en edades más avanzadas, entendemos que conocer la edad de inicio con exactitud nos permitirá desde el ámbito profesional que nos desempeña laboralmente poner remedio y énfasis en su erradicación.

\section{Material y Métodos}

En este apartado de la investigación se indican los elementos, instrumentos y muestra necesarios para elaborar este estudio que presenta un diseño de carácter exploratorio, descriptivo y de corte transversal.

\section{Participantes}

Tomaron parte en esta investigación 2.134 adolescentes españoles, de ambos sexos (49,8\% de hombres y 50,2\% de mujeres), con un rango de edad entre los 15 y 17 años $(\mathrm{M}=15,93$ años; $\mathrm{DT}=0,853)$ y matriculados en Segundo Ciclo de la Educación Secundaria Obligatoria (ESO). La muestra es representativa en cuanto al número de adolescentes analizados que se encuentran cursando este Ciclo de Educación Obligatoria en la provincia de Granada $(n=18.930)$, asumiendo un error muestral del 0.02 , con un nivel de confianza del 95\%, la muestra requerida fue de 2.231 participantes. Los participantes fueron seleccionados mediante un muestreo aleatorio por conglomerados por centros educativos y localidades. Debemos señalar que se detectaron 68 cuestionarios erróneos en su realización que fueron excluidos del estudio; y como 283 estudiantes no remitieron su consentimiento informado, por lo que no tomaron parte en este estudio.

\section{Variables e instrumentos}

En este estudio de investigación se tomó como referencia las siguientes variables e instrumentos:

- Género, según sea masculino o femenino.

- Autoconcepto, se recoge del cuestionario original "Autoconcepto Forma- 5 (AF5)" de García y Musitu (1999), y mide las dimensiones de Autoconcepto Académico (AA), Autoconcepto Social (AS), Autoconcepto Emocional (AE), Autoconcepto Familiar (AFM) y Autoconcepto Físico (AF). Este test consta de 30 preguntas, que se valoran con una escala Likert de cinco opciones, donde el 1 es Nunca y el 5 es Siempre; para cada dimensión se establece el sumatorio de los siguientes ítems (AA: ítems 1,6,11,16,21,26; AS: ítems 2,7,12,17,22,27; AE: ítems 3,8,13,18,23,28; AFM: ítems 4,9,14,19,24,29; AF: ítems 5,10,15,20,25,30). En el 
estudio de García y Musitu (1999) se determinó una fiabilidad de $\alpha=.810$, valor casi idéntico al detectado en nuestro trabajo (alpha de Cronbach de $\alpha=.833$ ); por dimensiones (AA: $\alpha=.773$; AS: $\alpha=.702$; AE: $\alpha=.697$; AFM: $\alpha=.778$; AF: $\alpha=.721$ ), los valores en todos los grupos eran satisfactorios al igual que plantearon en sus estudios Estévez, Martínez y Musitu (2006) o Cava et al., (2008).

- Escala de Consumo de Tabaco, se recoge del cuestionario original "Fagerström Test for Nicotine Dependence (FTND)" de Heatherton, Kozlowski, Frecker y Fagerström (1991), y traducido al castellano por Villareal- González (2009), establece el numero o cantidad de cigarros, el impulso por fumar y la dependencia de nicotina que se tiene. Este test consta de 6 preguntas, de las cuales las 4 primeras son de tipo dicotómico $(0=\mathrm{No}$ y $1=\mathrm{Si})$, y los dos ítems restantes se valoran con una escala Likert de cuatro opciones, donde el 0 es Nunca y el 3 es Siempre; se establece la suma que oscila entre el 0 y el 10 , y se establecen cuatro categorías $(0=$ No fumador, de 1 a $4=$ Dependencia Baja, 5 a $6=$ Dependencia Moderada y >6= Dependencia Alta). En el estudio de Villareal-González (2009) se determinó una fiabilidad de $\alpha=.970$ similar a la que se detecta en nuestro trabajo $\alpha=.956$.

- Escala del Consumo de Alcohol, procede del cuestionario original "Alcohol Use Disorders Identification Test (AUDIT)" de Saunders, Aasland, Babor, De la Fuente y Grant (1993), y adaptado al español por Rubio (1998), consta de 10 preguntas, donde las 8 primeras cuestiones tienen cinco posibles respuestas donde el 0 es Nunca y el 4 es a Diario o Mas, y los dos ítems restantes se valoran con tres posibles opciones que puntúan $0-2$ y 4 ; se establece la suma que variará entre el 0 y el 40 , y señalar que las tres primeras preguntas hablan de la cantidad y frecuencia de consumo de alcohol, las preguntas 4-5 y 6 determinan la dependencia de consumo de alcohol y los ítems del 7 al 10 reportan consumo dañino o perjudicial. En el estudio de Rubio (1998) se determinó una fiabilidad de $\alpha=.800$ similar a la que se detecta en nuestro trabajo $\alpha=.842$.

\section{Procedimiento}

Desde la Facultad de Ciencias de la Educación de la Universidad de Granada (Área de Corporal), se solicitó la colaboración de los Centros Educativos de la Provincia de Granada seleccionados, a partir de un muestreo anteriormente mencionado; para ello la dirección de cada centro educativo fue informada sobre la naturaleza de la investigación y se solicitó la colaboración de sus estudiantes; también se les adjuntó un modelo de autorización destinado a los responsables legales de los adolescentes pidiéndoles su consentimiento informado, garantizando en todo momento el anonimato de la información. Los investigadores estuvieron presentes durante la recogida de los datos donde no detectó ninguna anormalidad a reseñar. El estudio cumplió con las normas éticas del Comité de Investigación y Declaración de Helsinki de 1975. 


\section{Análisis de los Datos}

Para el análisis de los datos se utilizó el programa estadístico software SPSS 22.0., se establecieron los descriptivos básicos mediante la utilización de medias y frecuencias y para el estudio relacional se emplearon ANOVA y tablas de contingencia.

\section{Resultados}

En cuanto al análisis de los datos y observando los descriptivos que se muestran en la Tabla I, los 2.134 adolescentes presentaban proporciones similares por género, eran no fumadores en su mayoría $(90,9 \% ; \mathrm{n}=1.939)$, en lo que respecta al consumo de alcohol, la cantidad y frecuencia era de no consumo en un $44,9 \%(\mathrm{n}=958)$, mientras que entre escaso y medio consumo lo señalan el 48,5\% de los adolescentes; la no dependencia del alcohol era valorada por el $79,4 \%(n=1695)$ y en la misma línea el $65,3 \%$ señaló no provocar ningún daño tras la ingesta de la misma mientras que el restante 34,7\% declaró mostrar actitudes dañinas tras la ingesta de estos productos.

El Autoconcepto (AF-5) obtuvo un valor medio de 3,62, y en lo que respecta a las dimensiones los datos mostraron que según la Escala Likert señalada con anterioridad, el Autoconcepto Familiar y Social presentaron valores medios en torno al 4 (M=4,09 y $\mathrm{M}=3,94)$; mientras que el resto de dimensiones se situaron entre cifras medias de 3,30 y 3,40 .

\begin{tabular}{c|c}
\hline \multicolumn{2}{c}{ Género } \\
\hline Masculino & $49,8 \%(\mathrm{n}=1.062)$ \\
\hline Femenino & $50,2 \%(\mathrm{n}=1.072)$ \\
\hline \multicolumn{2}{c}{ Consumo Tabaco (FTND) } \\
\hline No Fumador & $90,9 \%(\mathrm{n}=1.939)$ \\
\hline Dependencia Baja & $7,4 \%(\mathrm{n}=158)$ \\
\hline Dependencia Media & $0,9 \%(\mathrm{n}=19)$ \\
\hline Dependencia Alta & $0,8 \%(\mathrm{n}=18)$ \\
\hline
\end{tabular}

Tabla 1. Descriptivos de las variables 


\begin{tabular}{|c|c|c|}
\hline \multicolumn{3}{|c|}{ Consumo Alcohol (AUDIT) } \\
\hline \multirow{4}{*}{$\begin{array}{l}\text { Cantidad y } \\
\text { Frecuencia }\end{array}$} & No Consume & $44,9 \%(n=958)$ \\
\hline & Escaso Consumo & $25,4 \%(n=541)$ \\
\hline & Consumo Medio & $23,1 \%(n=494)$ \\
\hline & Consumo Alto & $6,6 \%(n=141)$ \\
\hline \multirow{4}{*}{ Dependencia } & No Dependencia & $79,4 \%(n=1.695)$ \\
\hline & Dependencia Baja & $13,4 \%(n=285)$ \\
\hline & Dependencia Media & $5,8 \%(\mathrm{n}=124)$ \\
\hline & Dependencia Alta & $1,4 \%(n=30)$ \\
\hline \multirow{4}{*}{$\begin{array}{c}\text { Consumo } \\
\text { Dañino }\end{array}$} & No Daño & $65,3 \%(n=1.394)$ \\
\hline & Daño Escaso & $17,8 \%(\mathrm{n}=379)$ \\
\hline & A Veces provoca Daño & $11,8 \%(n=251)$ \\
\hline & Siempre Daño & $5,2 \%(n=110)$ \\
\hline \multicolumn{3}{|c|}{ Autoconcepto General (AF-5) (M=3,62; D.T=.457) } \\
\hline \multicolumn{2}{|c|}{ Autoconcepto Académico } & $\mathrm{M}=3,36(\mathrm{D} \cdot \mathrm{T}=.793)$ \\
\hline \multicolumn{2}{|c|}{ Autoconcepto Social } & $\mathrm{M}=3,94$ (D.T=.695) \\
\hline \multicolumn{2}{|c|}{ Autoconcepto Emocional } & $\mathrm{M}=3,32(\mathrm{D} \cdot \mathrm{T}=.758)$ \\
\hline \multicolumn{2}{|c|}{ Autoconcepto Familiar } & $\mathrm{M}=4,09$ (D.T=.788) \\
\hline \multicolumn{2}{|c|}{ Autoconcepto Físico } & $\mathrm{M}=3,40(\mathrm{D} \cdot \mathrm{T}=.782)$ \\
\hline
\end{tabular}

Tabla 1. Descriptivos de las variables (cont.)

Como se desprende de la siguiente figura, la dimensión de tipo familiar es la más valorada mientras que el tipo emocional es el menor puntuado por los adolescentes.

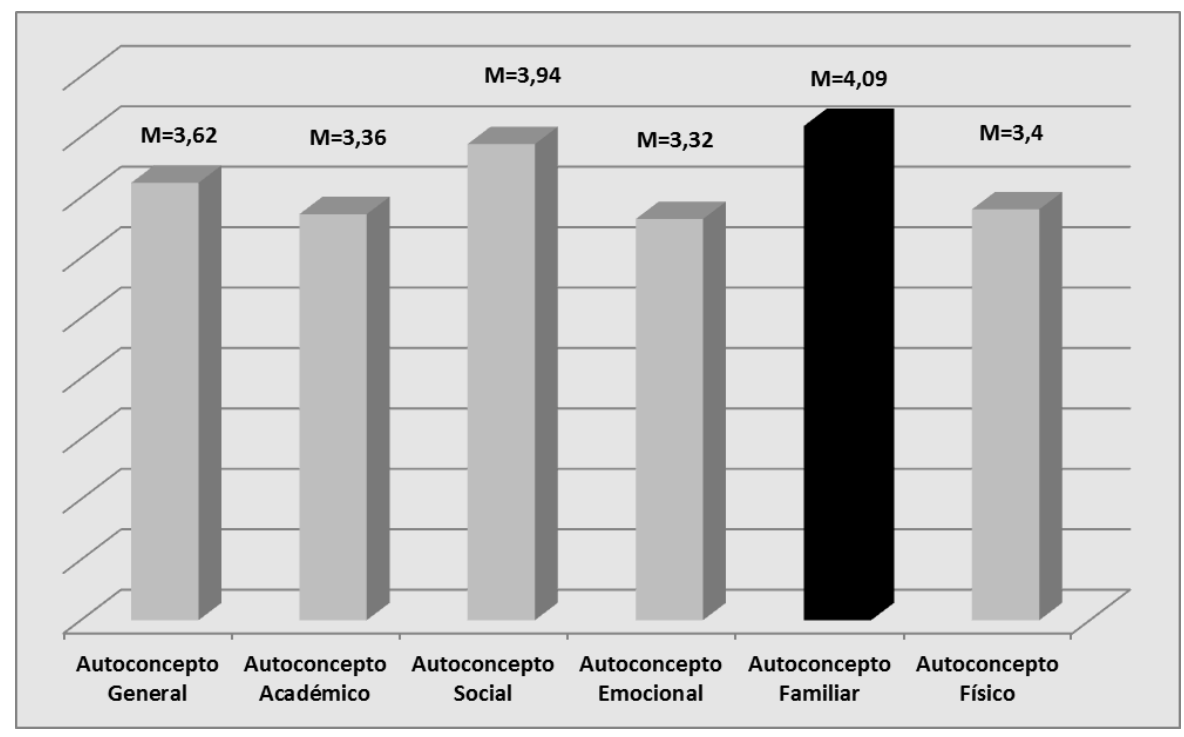

Figura 1. Autoconcepto General y Dimensiones del AF-5 
En cuanto a las relaciones de las dimensiones con el género, se detectan diferencias estadísticamente significativas $\left(\mathrm{p} \leq .05^{* *}\right)$ en la dimensión académica, emocional y física; en la primera de ellas las chicas presentan valores medios más elevados que los chicos; invirtiéndose este hecho en las dos restantes dimensiones donde no se halló asociación, como se aprecia en la siguiente tabla.

\begin{tabular}{|c|c|c|c|c|c|}
\hline \multicolumn{2}{|c|}{$\begin{array}{l}\text { Dimensiones } \\
\text { Autoconcepto }\end{array}$} & Media & $\begin{array}{c}\text { Desviación } \\
\text { Típica } \\
\end{array}$ & $\mathbf{F}$ & $X^{2}$ \\
\hline \multirow{2}{*}{ AA } & Masculino & 3,30 &, 730 & \multirow{2}{*}{15,674} & \multirow{2}{*}{$\mathrm{p}=.000 * *$} \\
\hline & Femenino & 3,42 & 742 & & \\
\hline \multirow{2}{*}{ AS } & Masculino & 3,95 & ,701 & \multirow{2}{*}{,252 } & \multirow{2}{*}{$\mathrm{p}=.616$} \\
\hline & Femenino & 3,93 & 689 & & \\
\hline \multirow{2}{*}{$\mathrm{AE}$} & Masculino & 3,46 &, 753 & \multirow{2}{*}{74,632} & \multirow{2}{*}{$\mathrm{p}=.000^{* *}$} \\
\hline & Femenino & 3,18 & 737 & & \\
\hline \multirow{2}{*}{ AFM } & Masculino & 4,11 &, 771 & \multirow{2}{*}{1,254} & \multirow{2}{*}{$\mathrm{p}=.263$} \\
\hline & Femenino & 4,07 &, 805 & & \\
\hline \multirow{2}{*}{$\mathrm{AF}$} & Masculino & 3,59 &, 763 & \multirow{2}{*}{127,040} & \multirow{2}{*}{$\mathrm{p}=.000^{* *}$} \\
\hline & Femenino & 3,21 &, 756 & & \\
\hline
\end{tabular}

Tabla 2. ANOVA de las dimensiones del Autoconcepto según Sexo.

En relación al consumo de tabaco y el autoconcepto los datos nos reportaron la asociación significativa $\left(\mathrm{p} \leq .05^{* *}\right)$, en cuanto a la dimensión académica, familiar y física; de esta forma en la primera y segunda los datos son bastante heterogéneos entre los no fumadores y los de dependencia media y alta; sin embargo en la de tipo físico, se aprecia la disparidad entre los no fumadores y los que presentan una alta necesidad de fumar cómo queda reflejado en la siguiente tabla (Tabla IV):

$1 * *$ Existen diferencias significativas $(\mathrm{P} \leq .05)$ 


\begin{tabular}{|c|c|c|c|c|c|}
\hline Dimen & es Autoconcepto & Media & $\begin{array}{c}\text { Desviación } \\
\text { Típica }\end{array}$ & $\mathbf{F}$ & $X^{2}$ \\
\hline \multirow{4}{*}{ AA } & No Fumador & 3,39 &, 734 & \multirow{4}{*}{15,824} & \multirow{4}{*}{$\mathrm{p}=.000 * *$} \\
\hline & Dependencia Baja & 3,01 & ,685 & & \\
\hline & Dependencia Media & 2,92 & ,778 & & \\
\hline & Dependencia Alta & 3,41 & ,703 & & \\
\hline \multirow{4}{*}{ AS } & No Fumador & 3,93 & ,690 & \multirow{4}{*}{2,259} & \multirow{4}{*}{$\mathrm{p}=.080$} \\
\hline & Dependencia Baja & 4,04 & ,716 & & \\
\hline & Dependencia Media & 4,21 & ,996 & & \\
\hline & Dependencia Alta & 3,86 & 664 & & \\
\hline \multirow{4}{*}{$\mathrm{AE}$} & No Fumador & 3,32 & ,752 & \multirow{4}{*}{1,625} & \multirow{4}{*}{$\mathrm{p}=.182$} \\
\hline & Dependencia Baja & 3,29 & ,796 & & \\
\hline & Dependencia Media & 3,67 & ,742 & & \\
\hline & Dependencia Alta & 3,46 & ,966 & & \\
\hline \multirow{4}{*}{ AFM } & No Fumador & 4,12 & ,770 & \multirow{4}{*}{11,542} & \multirow{4}{*}{$\mathrm{p}=.000 * *$} \\
\hline & Dependencia Baja & 3,78 & ,898 & & \\
\hline & Dependencia Media & 3,74 & 1,033 & & \\
\hline & Dependencia Alta & 3,82 & ,698 & & \\
\hline \multirow{4}{*}{$\mathrm{AF}$} & No Fumador & 3,42 & ,780 & \multirow{4}{*}{6,540} & \multirow{4}{*}{$\mathrm{p}=.000 * *$} \\
\hline & Dependencia Baja & 3,15 & ,765 & & \\
\hline & Dependencia Media & 3,57 & 699 & & \\
\hline & Dependencia Alta & 3,66 & ,766 & & \\
\hline \multirow{4}{*}{$\mathrm{AG}$} & No Fumador & 3,64 & 472 & \multirow{4}{*}{0,844} & \multirow{4}{*}{$\mathrm{p}=.842$} \\
\hline & Dependencia Baja & 3,45 & ,468 & & \\
\hline & Dependencia Media & 3,62 & ,554 & & \\
\hline & Dependencia Alta & 3,64 & ,435 & & \\
\hline
\end{tabular}

Tabla 3. ANOVA de relación del tabaco con AF-5.

En lo concerniente al análisis de los índices de consumo e ingesta de alcohol en cuanto a las cinco dimensiones de autoconcepto, en la siguiente tabla tras la realización de análisis ANOVA se muestra la asociación de las tres categorías del AUDIT con cada una de las dimensiones del AF5.

Debemos señalar que los datos del autoconcepto académico, social y familiar mostraron relación estadísticamente significativa en todos los casos $\left(\mathrm{p} \leq .05^{* *}\right)$ como se muestra en la tabla IV, por el contrario el autoconcepto físico no mostró en ningún caso asociación con las tres categorías propuestas en el AUDIT; puntualizar igualmente que en el caso del tipo emocional únicamente estableció relación al compararlo con el daño por el alcohol. 


\begin{tabular}{cccc}
\hline $\begin{array}{c}\text { Consumo Alcohol } \\
\text { (AUDIT) }\end{array}$ & $\begin{array}{c}\text { Dependencia del } \\
\text { Alcohol }\end{array}$ & $\begin{array}{c}\text { Cantidad y } \\
\text { Frecuencia }\end{array}$ & Daño Alcohol \\
\hline Autoconcepto Académico & $\mathrm{F}=26,846\left(\mathrm{p}=.000^{* *}\right)$ & $\mathrm{F}=52,621\left(\mathrm{p}=.000^{* *}\right)$ & $\begin{array}{c}\mathrm{F}=23,548 \\
\left(\mathrm{p}=.000^{* *}\right)\end{array}$ \\
\hline Autoconcepto Social & $\mathrm{F}=4,392\left(\mathrm{p}=.004^{* *}\right)$ & $\mathrm{F}=9,154\left(\mathrm{p}=.000^{* *}\right)$ & $\begin{array}{c}\mathrm{F}=7,482 \\
\left(\mathrm{p}=.000^{* *}\right)\end{array}$ \\
\hline Autoconcepto Emocional & $\mathrm{F}=2,167(\mathrm{p}=.090)$ & $\mathrm{F}=2,207(\mathrm{p}=.085)$ & $\begin{array}{c}\mathrm{F}=3,797 \\
\left(\mathrm{p}=.010^{* *}\right)\end{array}$ \\
\hline Autoconcepto Familiar & $\mathrm{F}=18,983\left(\mathrm{p}=.000^{* *}\right)$ & $\mathrm{F}=16,618\left(\mathrm{p}=.000^{* *}\right)$ & $\begin{array}{c}\mathrm{F}=16,431 \\
\left(\mathrm{p}=.000^{* *}\right)\end{array}$ \\
\hline Autoconcepto Físico & $\mathrm{F}=, 593(\mathrm{p}=.593)$ & $\mathrm{F}=1,335(\mathrm{p}=.261)$ & $\mathrm{F}=1,468(\mathrm{p}=.221)$ \\
\hline Autoconcepto General & $\mathrm{F}=1,697\left(\mathrm{p}=.000^{* *}\right)$ & $\mathrm{F}=1,153(\mathrm{p}=.165)$ & $\mathrm{F}=1,054(\mathrm{p}=.349)$ \\
\hline
\end{tabular}

Tabla 4. ANOVA del Consumo e ingesta de alcohol en relación con el AF5.

\section{Discusión y Conclusiones}

En el estudio realizado se determinó que casi la totalidad de los adolescentes no fumaban ni consumían alcohol de manera habitual, datos que siguen la línea de estudios previos como los de Rojas, Fleiz, Medina, Moron y Doménech (1999); Landero y Villarreal-González (2007); López-García, Esparza, Guzmán, Alonso y Rodríguez (2009); Salamó, Gras y Font. (2010) o Carballo et al., (2013), con porcentajes de ingesta de alcohol semejantes, sin embargo se constata que tres de cada diez estudiantes si lo consumían de forma esporádica (fines de semana) o habitual. Dichos adolescentes, comienzan a realizar salidas nocturnas los fines de semana y como exponen Martínez y Villar (2004) y Ruiz-Ruiseño, Ruiz-Juan y Zamarripa (2011), una de las causas influyentes en el mayor consumo de alcohol y tabaco en adolescentes españoles puede deberse al fenómeno "botellón" asentado desde hace décadas en nuestro país.

La principal explicación de los bajos porcentajes de consumo de ambas sustancias, es que la muestra estudiada es relativamente joven para presentar valores de consumo habituales altos, siendo la edad de 16 en adelante la que presenta porcentajes mayores en este campo. De ahí que los resultados deban interpretarse con cierta cautela, pues estos podrían deberse a que en estas edades es más habitual beber de forma esporádica, como los fines de semana y días festivos, que diariamente. De ahí que la mayoría de estudios muestren porcentajes elevados en cuanto a la ingesta de alcohol de forma eventual y, por el contrario, bajos en los que a dependencia se refiere.

En cualquier caso, se hace imprescindible la transmisión de hábitos familiares saludables por parte de la familia desde edades tempranas, ya que las probabilidades de aumento en consumo progresa conforme se incrementa la edad (Gómez, Fernández, Romero y Luengo, 2008). 
Por otra parte, los adolescentes mostraron tener unos niveles de autoconcepto global adecuados, obteniendo las dimensiones familiar y social resultados elevados frente al resto de áreas, lo cual indica que a estas edades se le concede mayor importancia a las relaciones familiares y al grupo de iguales. Estos resultados ratifican lo encontrado por otros autores, que destacan que un autoconcepto global adecuado favorece la consecución de un buen ajuste psicológico, competencia personal y disminución de problemas comportamentales (Padilla, García y Suárez, 2010; Fuentes, García, Gracia y Lila, 2011).

A su vez, es necesario destacar que la dimensión de tipo familiar es la más valorada por la muestra, siendo la emocional la que menos. Resultados similares y convergentes muestran Padilla et al., (2010) en lo que se refiere a la dimensión familiar; aunque se deben tratar con cierta cautela siguiendo lo expuesto por Malo, Bataller, Casas, Gras y González (2011) que observaron que el autoconcepto familiar desciende conforme el adolescente promociona cursos en la Educación Secundaria Obligatoria, volviendo a aumentar en bachillerato. Se podría pensar, partiendo de estos resultados, que en estas edades el pubescente es todavía dependiente de sus progenitores, por lo que existe un fuerte vínculo familiar.

En cuanto al ámbito emocional nos hallamos en la misma postura que Salum-Fares, Marín y Reyes (2011), los cuales obtuvieron como resultado de su análisis bajos niveles en este componente. Resultados divergentes encontraron Padilla et al., (2010) y Veliz y Apodaca (2012), entendemos que la obtención de dichos resultados puede deberse a que el alumnado encuestado se encuentra en una edad donde su personalidad se está configurando y sufren muchos altibajos emocionales, no estando seguros completamente de sí mismos. Tales resultados podrían sugerir la necesidad de impulsar esta variable, generando un clima cálido y participativo donde la aportación de cada alumno sea valorada para su mejora.

En cuanto a las relaciones de las dimensiones del AF-5 con el género, se detectan diferencias en la dimensión académica, emocional y física. En la primera de ellas, las chicas presentan valores medios más elevados que los chicos, resultados que son convergentes con los obtenidos por Padilla et al., (2010); Malo et al., (2011). Sin embargo, Backes (1994) y Gordillo et al., (2011) hallaron puntuaciones más bajas en el área académica por parte de las chicas. Tenemos la creencia que esta diferencia entre chicos y chicas en el área académica puede deberse a que la valoración global de la misma, enmascare diferencias en ciertas habilidades, por lo que sería interesante desglosar la competencia académica en varios sectores (Stevenson y Newman, 1986).

En lo que respecta al área emocional, un altísimo porcentaje de estudios sitúan a los chicos con resultados más positivos que sus compañeras, tal y como lo demuestran Padilla et al., (2010) y Veliz y Apodaca (2012). Éstas diferencias indican que las chicas son más propensas a padecer ansiedad, mientras que los chicos presentan una mayor adaptación personal, además de encontrarse en planos emocionales distintos por las diferencias en el desarrollo madurativo.

En cuanto al área física, la mayoría de los autores han encontrado diferencias de género, obteniendo los resultados más elevados los chicos (Pastor, Balaguer y 
Benavides, 2002; Ruíz de Azúa, Rodríguez y Goñi, 2005; Padilla et al., 2010; Malo et al., 2011; Gordillo et al., 2011). Por el contrario existen muy pocas investigaciones en las que los resultados se muestren a la inversa, es decir, chicas con un autoconcepto físico más elevado que los chicos (López-Justicia y Pichardo, 2003), aunque hay que destacar que los autores trabajan con pubescentes con visión afectada. Desde nuestro punto de vista, el hecho de que las chicas muestren un autoconcepto físico más bajo, se debe a que éstas son más estrictas con su imagen en edad adolescente, en comparación con los chicos, concediéndole más importancia al aspecto físico. Las chicas suelen madurar antes que los chicos y ello es un aspecto a tener en cuenta en las investigaciones centradas en este tema.

En lo referente al consumo de sustancias nocivas y su relación con el autoconcepto, los resultados nos indican que no existen apenas diferencias entre los fumadores y no fumadores en cuanto a la dimensión académica y familiar. Estos resultados no se muestran afines con los descubiertos por Moreno-Murcia, Moreno y Cervelló (2009) y Fuentes et al., (2011), los cuales afirman que estas dos dimensiones, junto con la física, se relacionan inversamente con el consumo de sustancias nocivas.

Por el contrario, en la dimensión física existen diferencias en cuanto al autoconcepto percibido por fumadores y no consumidores, teniendo estos últimos valores más elevados, tal y como indican Oliva et al., (2008). Sin embargo, Moreno, Muñoz, Pérez y Sánchez-Queija (2005) matizan que el consumo en la adolescencia no se relaciona con problemas de comportamiento y autoconcepto.

Por último, centrándonos en el consumo de alcohol, hemos de decir que las dimensiones académica, social, emocional y familiar se ven influenciadas con la ingesta de este tipo de bebidas. Al hilo de estos datos autores como Musitu et al., (2007), Cava et al., (2008) y Moreno-Murcia et al., (2009) apoyan tal consistencia, aunque éstos últimos estiman que la dimensión social se ve incrementada en valores con la ingesta de alcohol, al ser una herramienta que favorece las relaciones entre iguales. No obstante, esta relación significativa entre el autoconcepto y la dimensión social desaparecía al controlar el efecto estadístico de las variables sexo y edad. Sin embargo, no se han hallado ningún tipo de relación entre consumo de alcohol y el área física.. Mencionados resultados coinciden parcialmente por los expuestos por Alcántara (2012), la cual manifiesta que existe relación entre consumo de alcohol y área física, pero no con la emocional.

La principal limitación del estudio ha sido la edad de los participantes y el haberlo focalizado en la provincia de Granada, si bien el haber analizado diversas comarcas de la misma, nos permite tener poblaciones heterogéneas; esto pone de manifiesto lo interesante de aumentar el ratio de análisis a provincias limítrofes o a otras comunidades autónomas.

En conclusión podemos señalar que los adolescentes de segundo ciclo de Secundaria son en su mayoría no fumadores y el consumo de alcohol era perceptible únicamente en tres de cada diez jóvenes; asimismo los adolescentes presentaron cifras globales de autoconcepto normales, destacando principalmente el área familiar y social. En los jóvenes no se encontró asociación entre el consumo o ingesta de tabaco y 
alcohol y el autoconcepto en la mayor parte de las dimensiones, exceptuando aquellas procedentes del daño del alcohol, si bien eran muy escasos los sujetos encuadrados en este grupo por lo que se debe tener cautela con las cifras obtenidas.

Señalar que las hipótesis primera y segunda no se cumplen entendemos que propiciado por la edad de la muestra analizada y que la tercera únicamente se cumple cuando citamos el contexto social.

Estos hallazgos respaldan la necesidad de seguir profundizando en la investigación de hábitos saludables y de personalidad en los adolescentes, con el objetivo de detectar la etapa clave donde se generan los comportamientos nocivos que afectan a los jóvenes y que repercuten nocivamente en edades superiores. Parece incuestionable la necesidad de determinar los factores antecedentes así como elaborar programas encaminados a la disminución de conductas anómalas tanto familiares como académicas en etapas anteriores.

\section{Referencias bibliográficas}

ALCÁNTARA, S. A. (2012). Control conductual percibido, intención y autoconcepto para evitar el consumo de drogas en estudiantes de preparatoria. Tesis Doctoral: Universidad Autónoma de Nuevo León.

BACKES, J.S. (1994). Bridging the Gender Gap: Self-Concept in the Middle Grades. Schools in the middle, 3, 19-23.

CARBALlO, J.L., MARÍN, M., JÁUREGUI, V., GARCÍA, G., ESPADA, J., ORGILÉS, M. Y PIQUERAS, J.A. (2013). Consumo excesivo de alcohol y rendimiento cognitivo en estudiantes de secundaria de la provincia de Alicante. Health and Addictions: Salud y Drogas, 13(2), 157-163.

CAVA, M.J., MURGUI, S. Y MUSITU, G. (2008). Diferencias en factores de protección del consumo de sustancias en la adolescencia temprana y media. Psicothema, 20(3), 389-395.

CONTRERAS, O. R., FERNÁNDEZ, J. G., GARCÍA, L. M., PALOU, P. Y PONSETI, J. (2010). El autoconcepto físico y su relación con la práctica deportiva en estudiantes adolescentes. Revista de Psicología del Deporte, 19(1), 22-39.

COX, R. H. (2009). Psicología del Deporte: conceptos y sus aplicaciones. Madrid: Panamericana.

ECHEBURÚA, E. Y CORRAL, P. (2010). Adicción a las nuevas tecnologías y a las redes sociales en jóvenes: Un nuevo reto. Adicciones, 22, 91-96.

ESNAOLA, I., GOÑI, A. Y MADARIAGA, J. M. (2008). El autoconcepto: perspectivas de investigación. Revista de Psicodidáctica, 13(1), 179-194.

ESNAOLA, I., RODRÍGUEZ, A., Y GOÑI, E. (2011). Propiedades psicométricas del cuestionario de Autoconcepto AF5. Anales de Psicología, 27(1), 109-117. 
ESTÉVEZ, E., HERRERO, J., MARTÍNEZ, B. Y MUSITU, G. (2006). Agressive and non-agressive rejected: an analysis of their differences. Psychology in the Schools, 43, 387-400.

ESTÉVEZ, E., MARTÍNEZ, B. Y MUSITU, G. (2006). La autoestima en adolescentes agresores y victimas en la escuela: la perspectiva multidimensional. Intervención Psicosocial, 15, 223-232.

FUENTES, M. C., GARCÍA, F., GRACIA, E. Y LILA, M. (2011). Autoconcepto y ajuste psicosocial en la adolescencia. Psicothema, 23, 7-12.

GARCÍA, F. Y MUSITU, G. (1999). AF5: Autoconcepto Forma 5. Madrid: TEA Ediciones.

GÓMEZ, J.A., FERNÁNDEZ, N., ROMERO, E. Y LUENGO, A. (2008). El botellón y el consumo de alcohol y otras drogas en la juventud. Psicothema, 20(2), 211-217.

GONZÁLEZ, J. Y PORTOLÉS, A. (2014). Actividad física extraescolar: relaciones con la motivación educativa, rendimiento académico y conductas asociadas a la salud. Revista Iberoamericana de Psicología del Ejercicio y el Deporte, 9(1), 5165.

GOÑI, E. E INFANTE, G. (2010). Actividad físico-deportiva, autoconcepto físico y satisfacción con la vida. European Journal of Education and Psychology, 3(2), 199208.

GORDILlO, M., VICENTE, F., SÁNCHEZ, S., GÓMEZ, M. Y GORDILlO, T. (2011). Diferencias en el autoconcepto de adolescentes extremeños en función del género, el nivel socioeconómico-cultural y la población de pertenencia. Campo Abierto, 30(2), 65-67.

HEATHERTON, T., KOZLOWSKI, L., FRECKER R. Y FAGERSTRÖM, K.O. (1991). The Fagerström Test of Nicotine Dependence: a revision of the Fagerström Tolerance Questionnaire. British Journal of Addiction, 86(9), 1119-1127.

JIMÉNEZ, T.I., MUSITU, G., MURGUI, S. Y LEHALLE, H. (2007). Le role de la comunication familiale et de léstime de soi dans la delinquance adeolescente. Revue Internationale de Psychologie Sociale, 20(2), 5-26.

JIMÉNEZ, T.I., MUSITU, G., RAMOS, M.J. Y MURGUI, S. (2009). Community involvement and victimization at school: An analysis through family, personal and social adjustment. Journal of Community Psychology, 37(8), 959-974.

KAVAS, A. B. (2009). Self-esteem and health-risk behaviours among Turkish late adolescents. Adolescence, 44(173), 187-198.

KOKKEVI, A., RICHARDSON, C., FLORESCU, S., KUZMAN, M. Y STERGAR, E. (2007). Psychosocial correlates of substance use in adolescence: A cross-national study in six European countries. Drug and Alcohol Dependence, 86, 67-74. 
LANDERO, R. Y VILLARREAL-GONZÁLEZ, M.E. (2007). Consumo de alcohol en estudiantes en relación con el consumo familiar y de los amigos. Psicología y Salud, 17(1), 17-23.

LONDOÑO, C. (2010). Resistencia de la presión de grupo, creencias acerca del consumo y consumo de alcohol en universitarios. Anales de Psicología, 26(1), 2733.

LÓPEZ-GARCÍA, K.S., ESPARZA, S.E., GUZMÁN, F.R., ALONSO, M.M. Y RODRÍGUEZ, L. (2009). Expectativas del consumo de alcohol y tabaco en adolescentes escolares. Revista Científica Electrónica de Psicología, 7, 112-126.

LÓPEZ-JUSTICIA, M. D. Y PICHARDO, M. C. (2003). Diferencias de género en el autoconcepto de jóvenes afectados de baja visión. Revista de Educación, 330, 373384.

MALO, S., BATALLER, S., CASAS, F., GRAS, M. E., Y GONZÁLEZ, M. (2011). Análisis psicométrico de la escala multidimensional de autoconcepto AF5 en una muestra de adolescentes y adultos de Cataluña. Psicothema, 2011, 23(4), 871-878.

MARTÍNEZ, G. Y VILLAR, M. (2004). Estudio descriptivo del uso de drogas en adolescentes de educación media superior de Ciudad de Monterrey. Revista Latinoamericana Enfermagem, 12, 391 -397.

MORENO, C., MUÑOZ, V., PÉREZ, P., Y SÁNCHEZ-QUEIJA, I. (2005). Los adolescentes españoles y su salud. Resumen del estudio "Health Behaviour in School Aged Children (HBSC-2002)'. Madrid: Ministerio de Sanidad y Consumo.

MORENO-MURCIA, J. A., MORENO, R. Y CERVELLÓ, E. (2009). Relación del autoconcepto físico con las conductas de consumo de alcohol y tabaco en adolescentes. Adicciones, 21, 147-154.

MUSITU, G. Y GARCÍA, F. (2004). Consecuencias de la socialización familiar en la cultura española. Psicothema, 16, 288-293.

MUSITU, G., JIMÉNEZ, T. I. y Murgui, S. (2007). Funcionamiento familiar, autoestima y consumo de sustancias: Un modelo de mediación. Revista de Salud Pública de México, 49, 3-10.

OLIVA, A., PARRA, Á. Y SÁNCHEZ-QUEIJA, I. (2008). Consumo de sustancias durante la adolescencia: Trayectorias evolutivas y consecuencias para el ajuste psicológico. International Journal of Clinical and Health Psychology, 8, 153-169.

PADILLA, M.T., GARCÍA, S. Y SUÁREZ, M. (2010). Diferencias de género en el autoconcepto general y académicos de estudiantes de $4^{\circ}$ de E.S.O. Revista de Educación, 352, 495-515.

PASTOR, Y., BALAGUER, I. y Benavides, G. (2002). Influencia de los dominios del autoconcepto en la autoestima de los adolescentes. Revista de Psicología Aplicada, $12(3), 97-112$. 
PAZ-NAVARRO, L. D., ROLDÁN, R., Y GONZÁLEZ, M. (2009). Funcionamiento familiar de alumnos con bajo rendimiento escolar y su comparación con un grupo de rendimiento promedio en una preparatoria de la universidad de Guadalajara. Revista de Educación y Desarrollo, 10, 5-15.

PRADO, G. Y PANTIN, H. (2011). Reducing substance use and HIV health disparities among Hispanic youth in the U.S.A.: The Familias Unidas program of research. Psychosocial Intervention, 20, 63-73.

REIGAL, R., VIDERA, A. PARRA, J.L. Y JUÁREZ, R. (2012). Actividad físico deportiva, autoconcepto físico y bienestar psicológico en la adolescencia. Retos, Nuevas Tendencias en Educación Física, Deporte y Recreación, 22, 19-23.

ROJAS, E., FLEIZ, C., MEDINA, M., MORÓN, M. Y DOMÉNECH, M. (1999). Consumo de alcohol y drogas en estudiantes de Pachuca (Hidalgo). Revista de Salud Pública de México, 41(4), 297-308.

RUBIO, G. (1998). Validación de la prueba para la identificación de trastornos por el uso de alcohol (AUDIT) en Atención Primaria. Revista Clínica Especializada, 198, 11-14.

RUIZ DE AZÚA, S., RODRÍGUEZ, A. Y GOÑI, A. (2005). Variables socioculturales en la construcción del autoconcepto físico. Cultura y Educación, 17 (3), 225-238.

RUIZ-JUAN, F. Y RUIZ-RISUEÑO, J. (2011). Variables predictoras de consumo de alcohol entre adolescentes españoles. Anales de Psicología, 27(2), 350-359.

RUIZ-JUAN, F., DE LA CRUZ, E. Y RUIZ-RISUEÑO, J., GARCÍA, M. (2008). Youth smoking patterns and leisure-time physical activity. Retos, Nuevas Tendencias en Educación Física, Deporte y Recreación, 14, 75-79.

RUIZ-RUISEÑO, J., RUIZ-JUAN, F. Y ZAMARRIPA J.I. (2011). Alcohol y tabaco en adolescentes españoles y mexicanos y su relación con la actividad físicodeportiva y la familia. Revista Panamericana Salud Publica. 31(3), 211-220.

SALAMÓ, A., GRAS, M. E. Y FONT, S. (2010). Patrones de consumo de alcohol en la adolescencia. Psicothema, 22 (2), 189-195.

SALUM-FARES, A., MARÍN, A. R., Y REYES, A. C. (2011). Relevancia de las dimensiones del Autoconcepto en estudiantes de Escuelas secundarias de Ciudad Victoria, Tamalulipas, México. Revista Electrónica de Psicología Iztacala, 14(2), 255-272.

SAUNDERS, J., AASLAND, O., BABOR, T., DE LA FUENTE, J. Y GRANT, M. (1993). Development of the Alcohol Use Disorders Identification Test (AUDIT): Who collaborative Project on early detection of persons with harmful alcohol consumption-II. Addiction, 88, 791-804.

SHAVELSON, R. J., HUBNER, J. J. Y STANTON, J. C. (1976). Self concept: Validation of construct interpretations. Review of Educational Research, 46(3), 407-441. 
SORIANO, J. A., SAMPASCUAL, G. Y NAVAS, L. (2010). El autoconcepto físico en la clase de Educación Física: un análisis centrado en el sexo. International Journal of Developmental and Educational Psychology. INFAD. Revista de Psicología, 3(1), 419-425.

STEVENSON, H.W., Y NEWMAN, R.S. (1986). Long-term prediction of achievement and attitudes in mathematics and reading. Child Development, 57, 646-659.

TÉllez, J., COTE, M., SAVOGAL, F., MARTíNEZ, E. Y CRUZ, U. (2003). Identificación de factores protectores en el uso de sustancias psicoactivas en estudiantes universitarios. Medicina, 51, 15-24.

VELIZ, A. Y APODACA, P. (2012). Niveles de autoconcepto, autoeficacia académica y bienestar psicológico en estudiantes universitarios de la ciudad de Temuco. Salud y Sociedad, 3(2), 131-150.

VILLAREAL-GONZÁLEZ, M.E. (2009). Un modelo estructural del consumo de drogas y conducta violenta en adolescentes escolarizados. Tesis doctoral: Universidad Autónoma de Nuevo León (México).

VILLARREAL-GONZÁLEZ, M.E., SÁNCHEZ, J.C. Y MUSITU, G. (2013). Análisis psicosocial del consumo de alcohol en adolescentes mexicanos. Universitas Psychologica, 12(3), 857-873.

ZAMBOAGA, B. L., SCHWARTZ, S. J., JARVIS, L. H. Y VAN TYNE, K. (2009). Acculturation and substance use among Hispanic early adolescents: Investigating the mediating roles of acculturative stress and self-esteem. The Journal of Primary Prevention, 30, 315-333. 


\section{Correspondencia con los autores}

José Ignacio ÁLVARO GONZÁLEZ

Facultad de Ciencias de la Educación

Campus de Cartuja s/n

18071 Granada

e-mail: nachoalvarog@hotmail.com

Félix ZURITA ORTEGA

Facultad de Ciencias de la Educación

Campus de Cartuja s/n

18071 Granada

e-mail: felixzo@ugr.es

Manuel CASTRO SÁNCHEZ

Facultad de Ciencias de la Educación

Campus de Cartuja s/n

18071 Granada

e-mail: manuel_cs87@hotmail.com

Asunción MARTÍNEZ MARTÍNEZ

Facultad de Ciencias de la Educación

Campus de Cartuja s/n

18071 Granada

e-mail: asuncionmm@ugr.es

Susana GARCÍA SÁNCHEZ

Facultad de Ciencias de la Educación

Campus de Cartuja s/n

18071 Granada

e-mail: s_garcia_s@hotmail.com 